\title{
Astronomy in Thailand
}

\author{
Busaba Kramer \\ National Astronomical Research Institute of Thailand, \\ Physics Building, Chiang Mai University, Chiang Mai 50200 Thailand \\ email: busaba@narit.or.th
}

\begin{abstract}
During the last few years, Thailand has seen a significant change in the way astronomical research and education is pursued in the country. The government has approved the establishment of the National Astronomical Research Institute of Thailand (NARIT) which aims to develop not only astronomical research but also astronomy education at all levels, both in formal and informal education. A framework of national key projects exists which includes national facilities, national collaborative research networks, teacher training and public outreach programmes. Examples of these programmes will be presented in this paper.
\end{abstract}

Keywords. Astronomy education, Thailand, astronomy development, developing country, NARIT

\section{From the past to present}

Astronomy has been one of the interests of the Kings of Siam (former name of Thailand) since the 1600s. The first astronomical observatory was built in 1685 during King Narai's Era. In the 1800s, King Rama IV studied western astronomical text books and precisely calculated the location of the total solar eclipse in 1868. Figure 1 shows the observatory and location of the total solar eclipse in Thailand in 1868 and the portrait of King Rama IV and his equipment. Apart from astronomy, he also brought modern scientific education to the kingdom, therefore he is considered as the "Father of Thai Science".

Modern theoretical research in astronomy only started in Thailand in the 1930s at Chulalongkorn University. One of a few observatories which has been fully utilized since the early days is Sirindhorn Observatory at Chiang Mai University, established in 1977 and equipped with 0.4-m Cassegrain telescope. Since 1996, the observatory also has a 0.5-m Cassegrain telescope, however the light pollution in the nearby Chiang Mai City has been too high to observe for research purposes. Today, observatories at several other universities have been established with 0.4-m class telescopes but not all of them are fully utilized due to the lack of personnel trained in astronomy. Indeed, there are less than 30 professional astronomers nationwide, most of them being university lecturers. Not all of them are still active in research. Thailand now has 26 well established universities with a faculty of Science and 41 newly established universities (transformed from teacher colleges in 2004).

In school education astronomy was mostly neglected until 2001 when astronomy was added to the National Science Curriculum with strong support from the astronomy community. However, as a result Thailand now faces a serious shortage in teachers being capable of teaching astronomy, so that teacher training has been an important aspect in the plans to further develop astronomy in Thailand.

In 2004, the government approved the establishment of the National Astronomical Research Institute of Thailand (NARIT), under the Ministry of Science and Technology. NARIT is a research- emphasis institute providing a collaborative network for developing and strengthening knowledge in astronomy, so as to meet international standards. The 

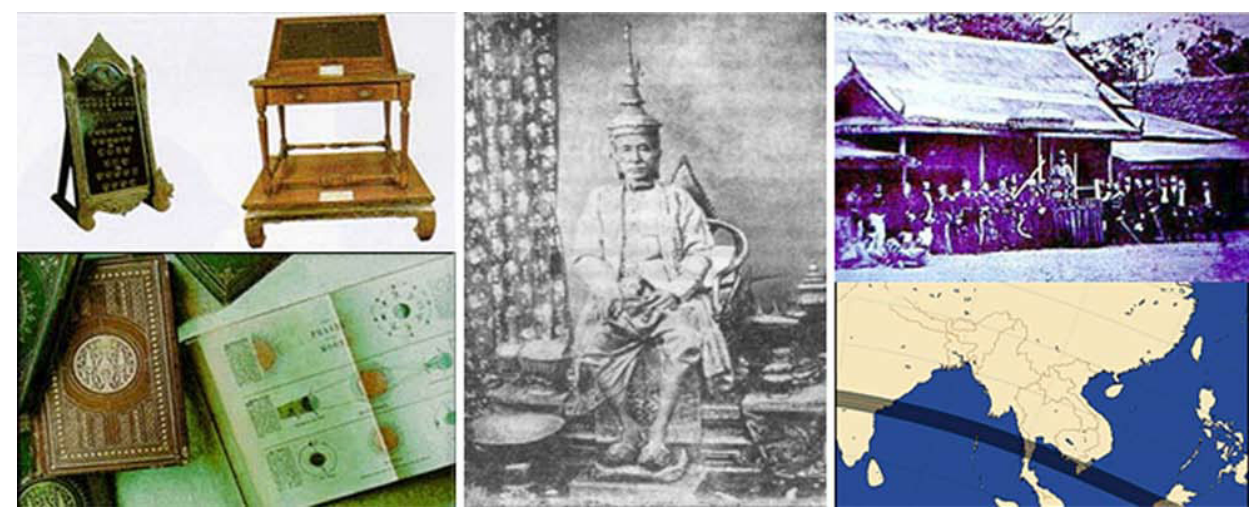

Figure 1. Centre - Image of King Rama IV; left - equipment and western astronomy textbooks which he used in calculating the eclipse path; right - group photo with the King at the observatory and map of the eclipse path in 1868 .

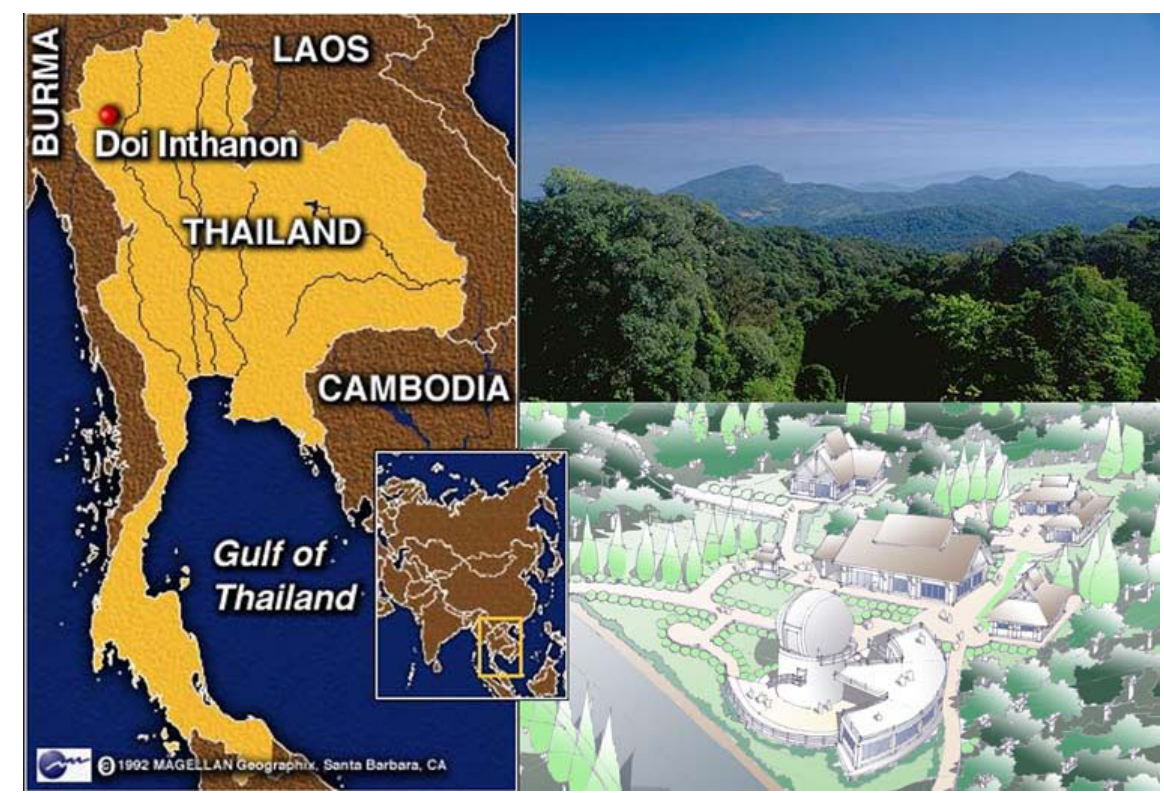

Figure 2. Thailand and locations of Doi Inthanon and National Observatory site.

institute also promotes education and learning culture in astronomy for the public and thus encourage them to seek further involvement with science and technology.

The main facility of NARIT is the National Observatory which is located on the very top of the highest mountain at the altitude of 2550 metres above the sea level, named Doi Intanon, in Chiang Mai province of northern Thailand, which is also renowned for the superb climate and tourist attractions. The National Observatory will operate a 2.4-metre reflecting telescope with an alt-azimuth drive system. This system has been synchronized with automatic motion of the dome structure, so that observation can be carried out efficiently. The National Observatory and the 2.4-metre reflecting telescope are under construction and are expected to be operated in the beginning of the year 2009. Figure 2 shows the conceptual design of the National Observatory and its location on the map of Thailand. 


\section{Roles of NARIT}

NARIT plays an important role in the development of astronomy in Thailand. Its aims are not only to establish the national facility but also to develop astronomical research and education in astronomy in Thailand on the national level, from school to advanced research.

\subsection{Astronomical research}

\subsubsection{National Policy}

Being an organization directly under the Ministry of Science and Technology, NARIT is directly involved in the national science policy for the development of astronomical research in Thailand. The institute exercises a 4-year rolling strategic plan (e.g. 20042008) which supports the National Science and Technology Policy.

\subsubsection{Infrastructure}

Infrastructure is crucial for the development of astronomical research. NARIT aims to establish the integrated infrastructure which will support the need of both research and technology transfer. These are the National Observatory, a research centre, data and IT centre, a Learning Centre for Astronomy, and Technical Support and Incubation Centre for Telescope Technologies.

\subsubsection{Human resources}

Human resources are one of the most important key factors for the development of Thailand as a country. One of the National Key Success Factors is therefore to increase the ratio of the number of scientific researchers in Thailand to the number of population to 8:10 000 within 10 years (i.e. by 2016). Therefore, with a rough estimate for the population of Thailand of $\sim 60$ million people, the target for the number of scientific researches in Thailand by 2016 is about 48000 . Estimating that about $2 \%$ of all researchers should work in the fields of astronomy, this implies a total of about 960 astronomers and astrophysicists. This is a very ambitious goal considering that this number should be reached in 10 years from a starting number of less than 30 astronomers. However, this number may indeed be required as a critical mass to self-sustain astronomical research and, in particular, to train a sufficient number of teachers for more than 40000 schools all over Thailand.

In order to reach this goal, NARIT is working on planning strategies in developing human resources for astronomical research in Thailand. The strategies are as the following:

(1) Allocating PhD Scholarships to study abroad. By working in world class astronomical institutes, students will gain the most advanced research knowledge and related technologies. Furthermore, after graduation and returning to Thailand, the young researchers will continue their link with the international organizations, therefore enabling them to pursue high quality research and high productivity while working back home.

(2) Creating Postdoctoral Research Fellowships and Senior Research Fellowships to recruit young and senior researchers from the international market. These human resources will help to improve the capability of producing $\mathrm{PhD}$ and MSc students in Thailand. Moreover, they will also create networks and links to the international astronomical community as well.

(3) Promoting MSc and $\mathrm{PhD}$ graduate courses among Thai universities. The $\mathrm{PhD}$ graduates will be fed in to the research community while the MSc graduates will be fed in to both the research community as research assistants and highly qualified school teachers. 
(40) Promoting a number of lectureship positions in astronomy at the universities which currently do not have astronomy courses, especially in the recently established (41) universities.

(5) Promoting the number of the nation's IAU individual members and the exchange of astronomers through a stop-over astronomers' programme and hosting astronomy meetings and workshops.

\subsubsection{Networking and collaborations}

Networks and collaborations are crucial to the development of astronomical research, both nationally and internationally. Within Thailand, universities will be encouraged to join as networks to improve their research and education capacity, while NARIT plays an important role in providing national facilities and facilitating the networks. One of the most needed outputs from the networks will be the $\mathrm{PhD}$ and $\mathrm{MSc}$ collaborative programmes.

NARIT also aims to host national and international meetings and workshops in astronomy. In March 2007, NARIT will host the annual astronomy conference, Thai National Astronomy Meeting 2007 (TNAM2007) in Chiang Mai. Since the number of astronomical research community in Thailand are not so high, this meeting will be held as part of the annual national physics conference. On the international level, the institute is now planning to host the first South-East Asia Astronomical Network Workshop (SEAAN2007). More information about the network can be found in Soonthornthum's paper in these proceedings. The institute is also planning to host the International Pulsar Workshop (http://www.nari.or.th/pulsar2007) in Krabi, during March 29 - April 7, 2007 and the $8^{\text {th }}$ Pacific Rim Conference on Stellar Astrophysics in Phuket in 2008.

\subsection{Education in astronomy}

The role of NARIT is not only in the development of astronomical research but also in education in astronomy in Thailand on the national Level The scope can be extended from all-age school education to the public understanding of science.

\subsubsection{National policy}

Similarly to the national policy for astronomical research, NARIT is also involved in the policy making for astronomical education by exercising a similar 4-year rolling strategic plan.

\subsubsection{NARIT outreach programme}

Despite being a newly established institute, NARIT has already carried out several outreach programmes since its first year. Example of the current outreach programmes are the following:

(1) Astronomy exhibitions - organized during the National Science Weeks in Bangkok which are hosted by the Ministry of Science and Technology annually. In these 12-day exhibitions, more than 300000 students attend from schools nationwide.

(2) Mobile exhibition units - containing posters, astronomical models, telescopes and equipment and learning material which can be transported to remote schools in other provinces.

(3) Online media and e-learning - the institute is working closely with several partners to develop online materials for school and public, see http://www.astroschool.in.th and http://www.stkc.go.th for example.

(4) Media and press - the institute is running regular radio shows and newspapers articles. 
(5) Family Astro-Sunday - under the government policy to encourage the family to spend time together during the weekend, the institute organizes star parties at the Sirindhorn Observatory on Sunday twice a month.

\subsubsection{Formal learning}

Astronomy has been included in National Science Curriculum since 2001. However, there is still a lack of personnel, knowledge and learning materials in astronomy. NARIT is working closely with the Institute for the Promotion of Teaching Science and Technology (IPST), Ministry of Education in the development of the above factors. The key projects include curriculum development, distance learning in astronomy, certified teacher training programmes, school and teacher networks, and promoting gifted and talented students in astronomy. During Nov 30 - Dec 9, 2007, NARIT together with the Promotion of Academic Olympiads and Development of Science Education Foundation (POSN) organizes the $1^{\text {st }}$ International Olympiad on Astronomy \& Astrophysics (IOAA) in Chiang Mai to promote interest and education in astronomy and astrophysics of high-school students.

\subsubsection{Informal learning}

Learning science outside school is one of the best ways to discover and to be inspired by science. NARIT aims to promote astronomy in informal learning in collaboration with the National Science Museum (NSM), an organization under Ministry of Science and Technology, and gives academic advice in astronomy to other science museums and planetarium nationwide. The institute also realizes the important role of amateur astronomers and astronomy clubs and societies in astronomy development in Thailand.

\section{Conclusions}

From the background of astronomy in Thailand from past to present, it can be said that now is a Golden Era for the development of astronomy in Thailand after NARIT has been established with strong supports from the government. While astronomy helps to promote scientific awareness of the community, industrial links and technology transfer are important also. Given the current need for of additional teachers, lecturers and researchers in astronomy, there are good opportunities for pursuing careers in astronomy in Thailand. Collaborations and networking are always important for the development. 


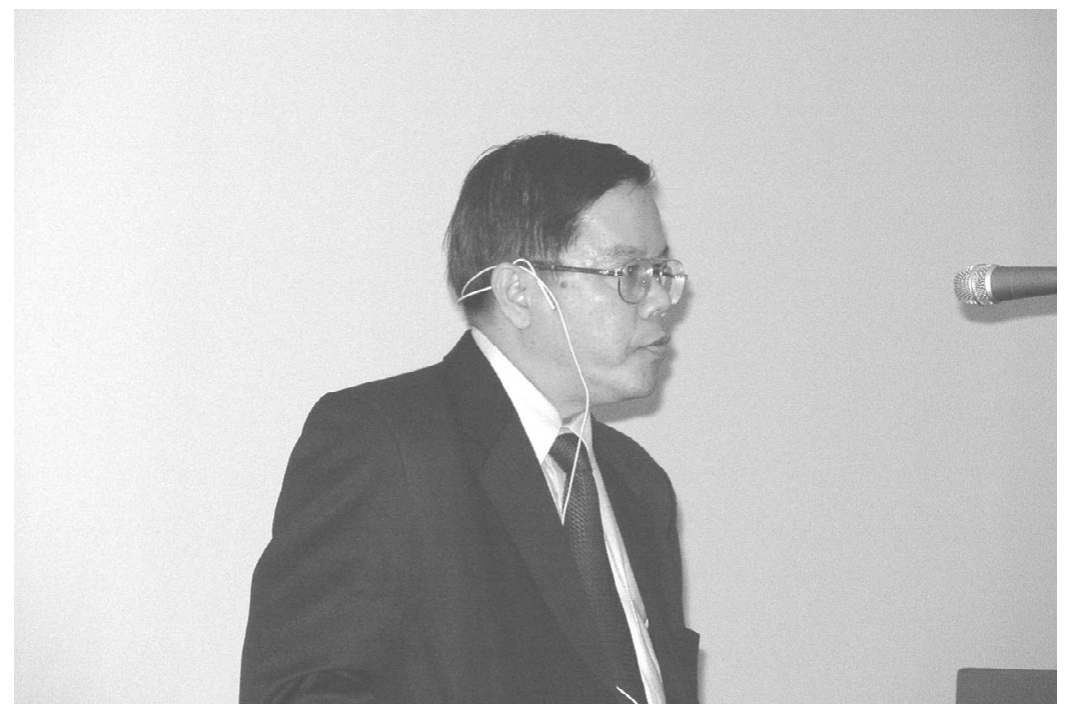

Boonrucksar Soonthornthum

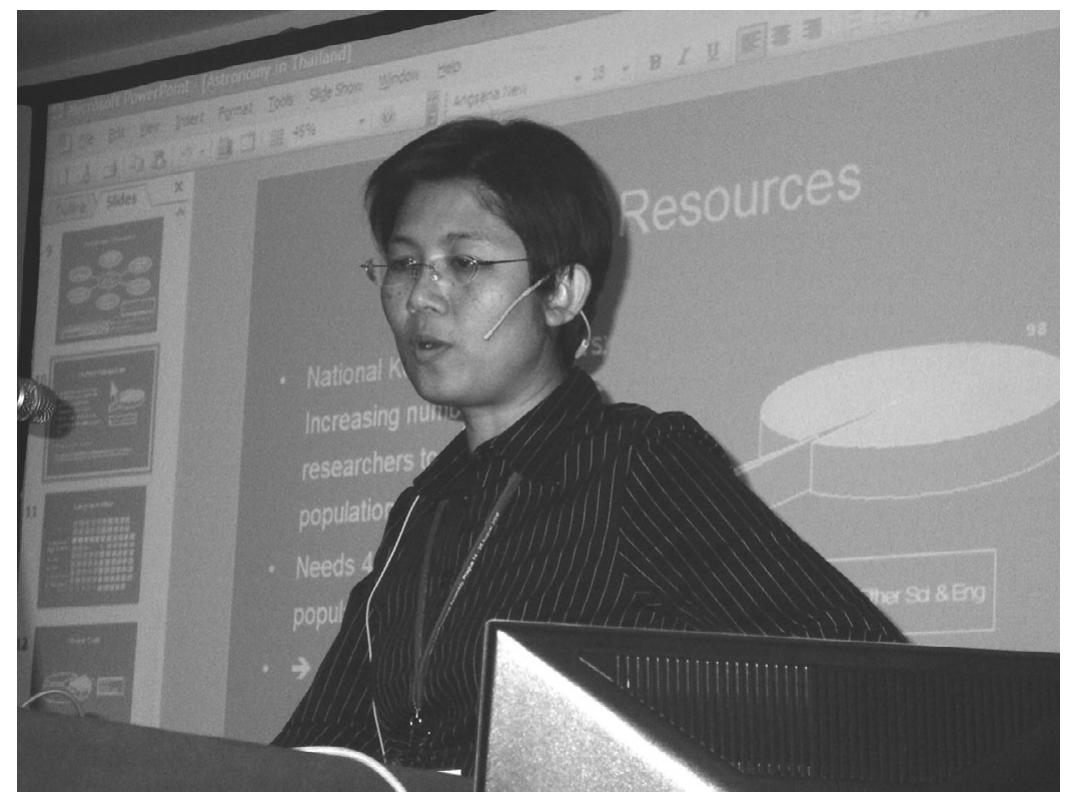

Busaba Kramer 